Title: Severe cutaneous eruptions following the topical use of preparations containing bufexamac: is it time to reconsider its registration in Australia?

Running Title: Cutaneous reactions after bufexamac use

Dr Adam G Harris ${ }^{1,2}$, Dr Samra L Saikal, Dr Jim Scurry ${ }^{3}$ Dr John Relic ${ }^{1}$, Associate Professor Rosemary L Nixon ${ }^{4}$, Dr Paul Chee ${ }^{1,2}$

${ }^{1}$ Department of Dermatology, John Hunter Hospital, Newcastle, New South Wales, Australia

${ }^{2}$ Faculty of Medicine, University of Newcastle, Newcastle, New South Wales, Australia ${ }^{3}$ Department of Anatomical Pathology, John Hunter Hospital, Newcastle, New South Wales, Australia

${ }^{4}$ Occupational Dermatology Research and Education Centre, Skin and Cancer Foundation Inc., Melbourne, Victoria, Australia

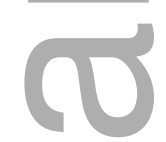

Corresponding Author: Adam Harris

Email: adamgeorgeharris@gmail.com

Phone: $\quad 0452523659$

Address: Department of Dermatology, Royal Newcastle Centre. Lookout Road New Lambton Heights

NSW 2305

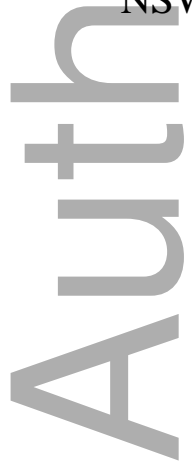

This is the author manuscript accepted for publication and has undergone full peer review but has not been through the copyediting, typesetting, pagination and proofreading process, which may lead to differences between this version and the Version of Record. Please cite this article as doi: $10.1111 /$ ajd.12910

This article is protected by copyright. All rights reserved 


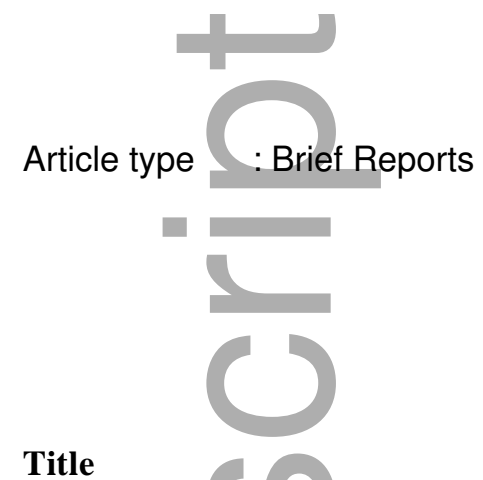

Severe cutaneous eruptions following the topical use of preparations containing bufexamac: is it time to reconsider its registration in Australia?

\begin{abstract}
Despite being a well-recognised cause of allergic contact dermatitis with an embargo in many countries around the world, bufexamac is available over the counter in topical preparations in Australia. We present a series of patients who developed severe cutaneous eruptions after the topical application of bufexamac containing preparations to highlight the potential risks of this medication, as well as advocate for the reconsideration of its registration by the Therapeutic Goods Administration in Australia.
\end{abstract}

Keywords: bufexamac, contact dermatitis, non-steroidal anti-inflammatory agents, patch tests, drug reaction

\title{
Learning Points:
}

- Bufexamac is a potent sensitiser available over the counter as a topical 'first aid' cream

- As well as allergic contact dermatitis, adverse reactions to bufexamac can present with polymorphic eruptions away from the site of application

- We encourage clinicians to assess for, diagnose and report bufexamac induced reactions

\section{Introduction}

Bufexamac is a non-steroidal anti-inflammatory drug (NSAID) available over the counter in Australia as a $5 \%$ topical preparation in combination with $1 \%$ lidocaine hydrochloride monohydrate and $0.1 \%$ chlorhexidine gluconate. This combination is registered with the Therapeutic Goods Administration (TGA) under various trade names (Table 1) for the treatment of 'insect bites, stings and itches; and minor cuts, 
abrasions, sunburn and burns', for which there is no published data demonstrating its efficacy [1]. Topical bufexamac is a well-recognised cause of allergic contact dermatitis (ACD) at the site of application as well as more widespread polymorphic cutaneous eruptions [2]. It is not available in New Zealand, Japan, the European Union (including the United Kingdom), the United States of America and Canada [2-5].

We present a series of patients who developed severe cutaneous eruptions after topical application of preparations containing bufexamac to highlight the potential risks, and advocate for reconsidering its registration by the TGA in Australia.

\section{Case series}

Patient One

A 7-year-old Caucasian female presented with a ten-day history of a pruritic erythematous eruption affecting her face, trunk, limbs and genitalia. In addition, she had oedema of her eyelids, and vesicles and bullae involving her nose, upper lip and palms (Figure 1). A cream containing bufexamac (Medi Quattro) had been applied to her abdomen one day prior to the commencement of the eruption. Blood tests showed a neutrophilia, and an eosinophilia of $3.9 \times 10^{9} / \mathrm{L}$. A skin biopsy showed parakeratosis, acanthosis, spongiosis and papillary dermal oedema with a moderate superficial perivascular lymphocytic infiltrate and eosinophils.

She was admitted to hospital and treated with potent topical corticosteroids under wet dressings, and oral antihistamines. While in hospital, she developed a secondary bacterial infection that was treated with oral and topical antibiotics (Figure 1). The eruption resolved with exfoliation. At one year of follow up she had no ongoing sequelae.

\section{Patient Two}

A 68-year-old Caucasian male presented with a ten-day history of a pruritic, erythematous and oedematous eruption affecting his trunk, limbs and genitalia (Figure 2). Urticated plaques, some of which were targetlike, were evident on the periphery of the eruption (Figure 2). A bufexamac containing cream (Medi Quattro) had been applied to his right arm three days prior to the development of the eruption. Blood tests showed a neutrophilia, and an eosinophilia of $0.8 \times 10^{9} / \mathrm{L}$. A skin biopsy showed similar features to patient one, but with more eosinophils.

He was treated as an outpatient with wet dressings, potent topical corticosteroids and oral prednisolone. The eruption also resolved with exfoliation, and at eight months follow up he showed no ongoing sequelae.

\section{Patient Three}

A 78-year-old Caucasian female presented to a rural emergency department with a six-day history of a pruritic, erythematous and oedematous eruption affecting her face, trunk and limbs. This began two days after using a cream containing bufexamac (Chemmart Antiseptic Cream). Blood tests and skin biopsies were 
not performed.

She was treated as an outpatient with wet dressings with potent topical corticosteroids and oral antihistamines. The eruption resolved over two months, and there were no ongoing sequelae at a threemonth follow up.

\section{Patch testing}

Following resolution of the eruption, patch testing was performed on all patients using the Australian Baseline Series (ABS), with the addition of chlorhexidine gluconate [6]. Patches were applied to the back for 48 hours and read at day two and four, where reactions were measured as irritant, doubtful (+/-), weak positive (1+), strong positive (2+) and extremely positive (3+).

Case one had a 3+reaction to bufexamac, a 2+ reaction to DMDM hydantoin and a 1+ reaction to nickel, colophony, fragrance mix and coconut diethanolamide. Case two had a 2+ reaction to bufexamac. Case three had a $2+$ reaction to bufexamac and lidocaine and a $1+$ reaction to hydroperoxides of limonene. In all three cases the reaction to bufexamac was strongly positive (Figure 3), and considered relevant to the patients' clinical presentation, given temporal association. Other positive reactions (i.e. to nickel) were deemed irrelevant given the lack of both exposure and temporal association with the patients' symptoms. The clinical history and matching patch test results allowed a diagnosis of ACD to bufexamac to be made in all three cases.

\section{Discussion}

In Australia, there have been 40 reports to the TGA of skin reactions attributed to topical use of preparations containing bufexamac. These reactions have included pruritus, dermatitis, oedema, urticaria, blistering and exfoliation [7]. Although significant, this is likely to only represent a snippet of the true morbidity caused by this medication. In addition to the patients presented above, we have consulted on multiple others with suspected cutaneous reactions to bufexamac who were not patch tested, and thus excluded from this series.

Topical bufexamac is well known to cause ACD and has been included in the ABS since its inception in 2015. An Australian study of 5281 patients estimated a sensitisation rate of $2 \%$ [6], whilst the largest study to date of 14,728 control patients patch tested in Germany, Austria and Switzerland estimated a sensitisation rate of $0.7 \%$ [8]. This lower rate most likely reflects the fact that bufexamac is only recommended for use in adults in Switzerland, and is not available in either Germany or Austria [5]. In addition to local reactions, generalised ACD, erythema multiforme-like reactions, acute generalised exanthematous pustulosis, pruritic pigmented dermatoses and a reaction imitating a baboon syndrome have been reported in the medical literature $[2,4,8,9]]$. In our series, all patients had $\mathrm{ACD}$ at the site of application, as well as reactions that extended well beyond the site. One case had erythema-multiforme like lesions at the periphery of the eruption. This polymorphic and atypical clinical presentation may obscure the correct diagnosis of bufexamac-induced $\mathrm{ACD}$, resulting in under-reporting of these reactions. 
There are no studies demonstrating the efficacy of topical bufexamac for any of the indications listed by the TGA. Of interest, the French Summary or Product Characteristics notes bufexamac containing preparations are contraindicated in patients with injured skin including wounds, burns, oozing or ulcerated lesions, conditions that are similar to TGA-approved indications [5]. Evidence for the efficacy of topical bufexamac in the treatment of inflammatory dermatoses is also lacking. The majority of the studies investigating this were performed in France in the 1970s, with the largest being a double blinded controlled trial of 193 participants with various forms of dermatitis. There was no improvement with topical bufexamac in comparison to the placebo [10]. Since that time, publications concerning bufexamac mostly report adverse events rather than any data relating to efficacy.

To the best of our knowledge, topical bufexamac has never been approved for use in the United States of America or Canada. In 2010, the European Medicines Agency's Committee for Medicinal Products for Human Use recommended that marketing authorisation for bufexamac containing preparations be revoked throughout the European Union (including the United Kingdom) [3]. In Japan pharmaceutical companies voluntarily recalled bufexamac containing preparations in 2010 [4]. In New Zealand, topical bufexamac has not been marketed since 2002, and in 2011, Medsafe, a business unit of the ministry of health recommended the revocation of consent to distribute bufexamac containing medicines in New Zealand [5].

\section{Recommendation}

Topical bufexamac has no published efficacy for TGA-listed indications, but clearly has significant cutaneous adverse effects, as we have highlighted. We therefore strongly encourage the TGA to reconsider its registration for use in Australia.

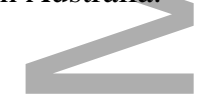

\section{Reference List}

1. Public Australian Register of Therapeutic Goods summaries of products containing bufexamac can be found at https://www.tga.gov.au/ (Current January 2018).

2. Pan Y, Nixon R. Allergic contact dermatitis to topical preparations of bufexamac. Australas J Dermatol 2012; 53(3): 207-210.

3. Uter W, Schnuch A. EMA revokes marketing authorization for bufexamac. Contact Dermatitis 2011; 64(4): $235-236$.

5. Medsafe New Zealand. Summary of data on the benefits and risks for bufexamac-containing medicines indicated for the relief of dermatitis, rash and hives from the minutes of the 145th Medicines Adverse Reactions Committee Meeting.

6. Toholka R, Wang YS, Tate B, et al. The first Australian Baseline Series: recommendations for patch testing in suspected contact dermatitis. Australas J Dermatol 2015; 56(2): 107-115.

7. Adverse events reports to the Therapeutic Goods Administration associated with products containing bufexamac can be found through The Database of Adverse Events Notifications at https://www.tga.gov.au/ (Current January 2018). 
8. Arikawa J, Okabe S, Kaneko T. Allergic contact dermatitis with spreading over extensive regions due to topical use of 5\% bufexamac ointment. J Dermatol 2004; 31(2): 136-138.

9. Fukuda H, Sato Y, Usami N, et al. Contact dermatitis caused by bufexamac sparing the eruption of herpes zoster. J Dermatol 2012; 39(4): 405-407.

10. Christiansen JV, Gadborg E., Kleiter I, et al. Efficacy of bufexamac (NFN) cream in skin diseases. A double-blind multicentre trial. Dermatologica 1977; 154(3): 177-184.

\section{Figure Legend}

Figure 1: Bullae on the palms after application of a bufexamac containing cream. The right hand developed a secondary bacterial infection.

Figure 2. Widespread cutaneous eruption after applying a bufexamac containing cream with target-like urticated plaques on the periphery.

Figure 3. Positive patch testing to topical bufexamac in cases one, two and three (left to right)

\section{Table 1}

\begin{tabular}{|l|}
\hline $\begin{array}{l}\text { Therapeutic Goods Administration: Topical } \\
\text { preparations Containing Bufexamac in Australia } \\
{[1]}\end{array}$ \\
\hline Amcal First Aid Cream tube \\
\hline Blooms The Chemist First Aid Cream tube \\
\hline Chemists' Own Antiseptic Plus cream tube \\
\hline Chemmart Antiseptic Cream tube \\
\hline Cipla Skin Assist First Aid Cream tube \\
\hline Medi Quattro First Aid Cream \\
\hline Pharmacist Formula Antiseptic Cream tube \\
\hline Pharmacy Action Antiseptic Cream tube \\
\hline
\end{tabular}




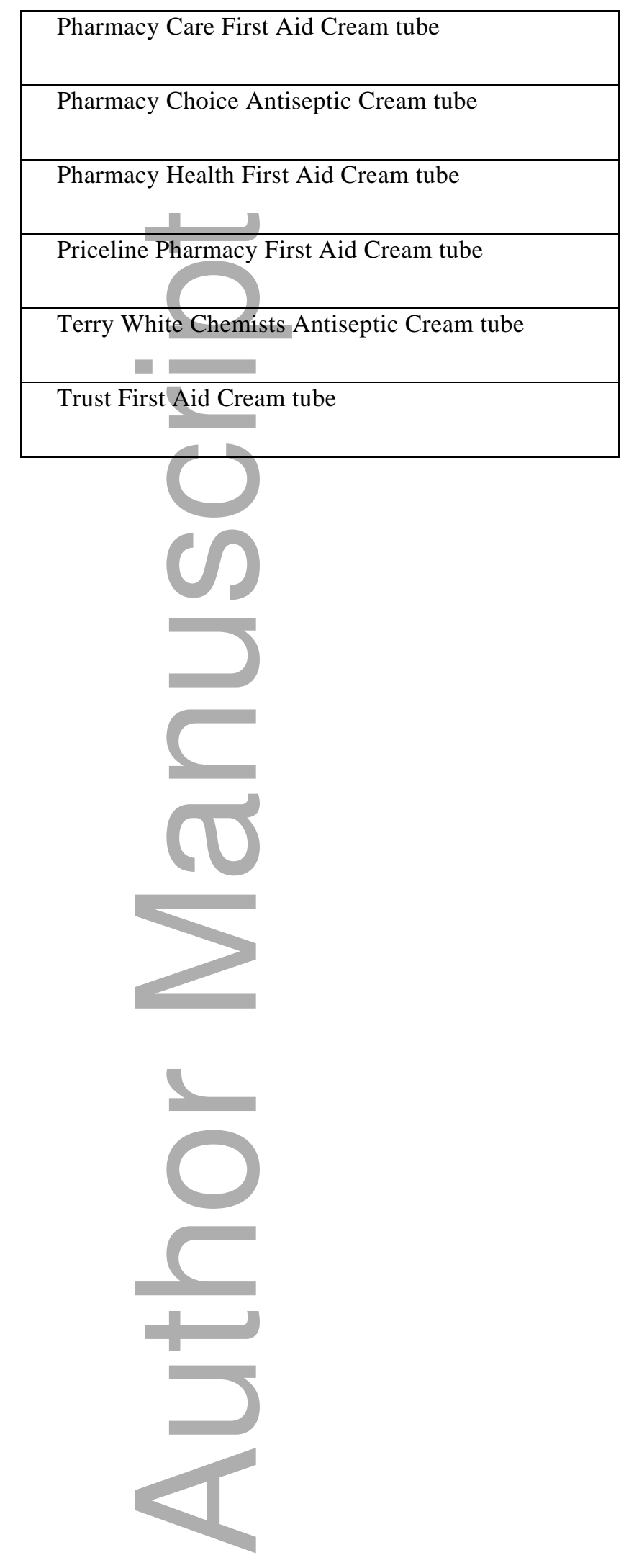

This article is protected by copyright. All rights reserved 


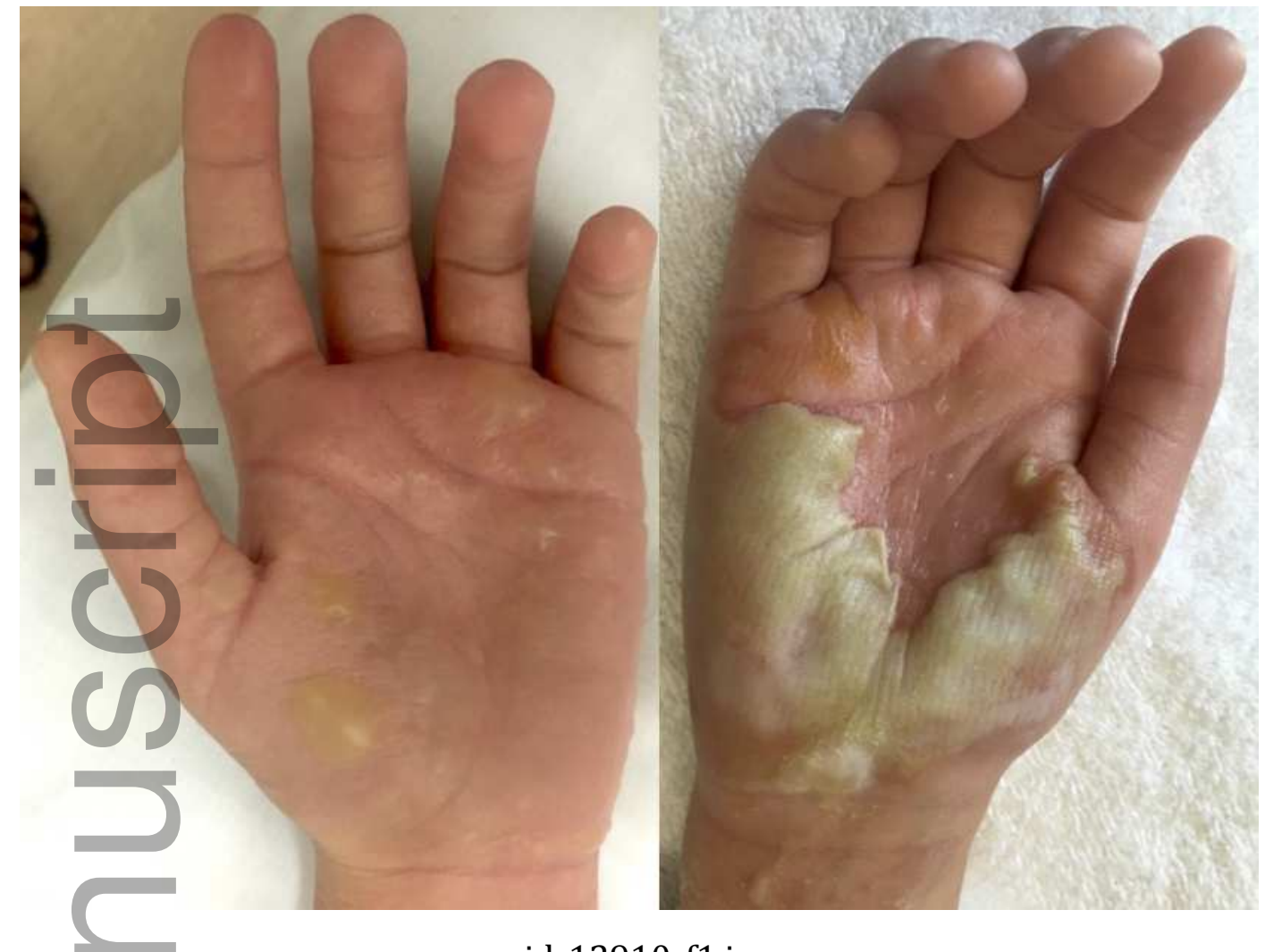

ajd_12910_f1.jpg
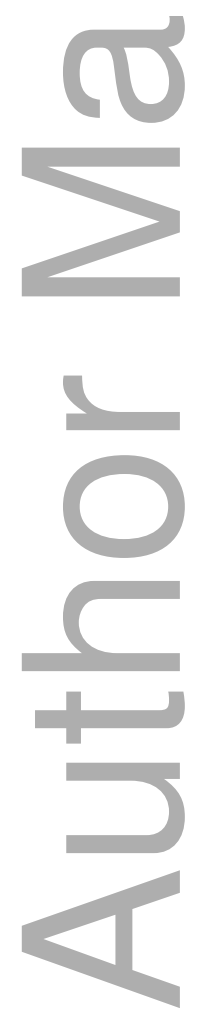

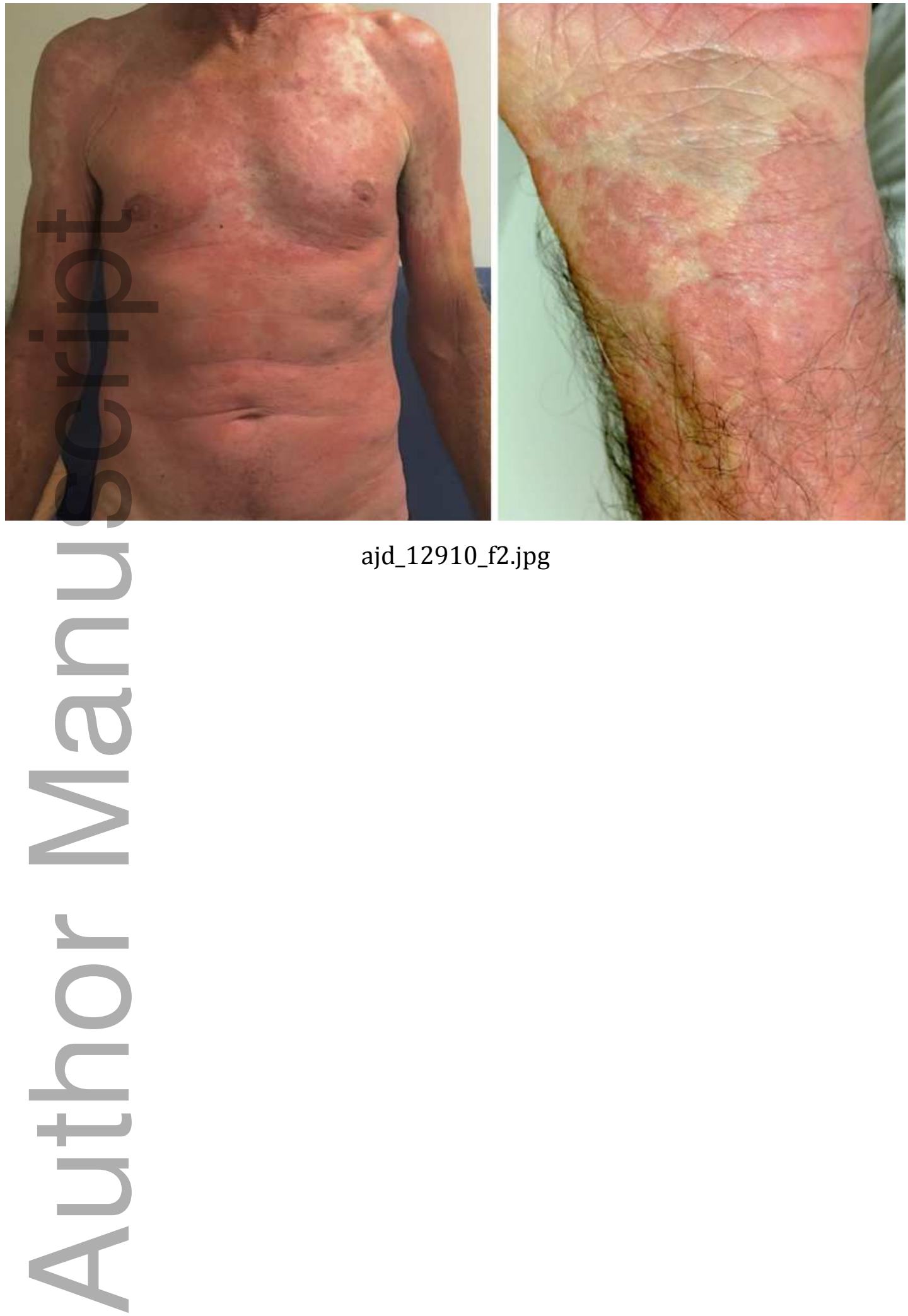

ajd_12910_f2.jpg

This article is protected by copyright. All rights reserved 


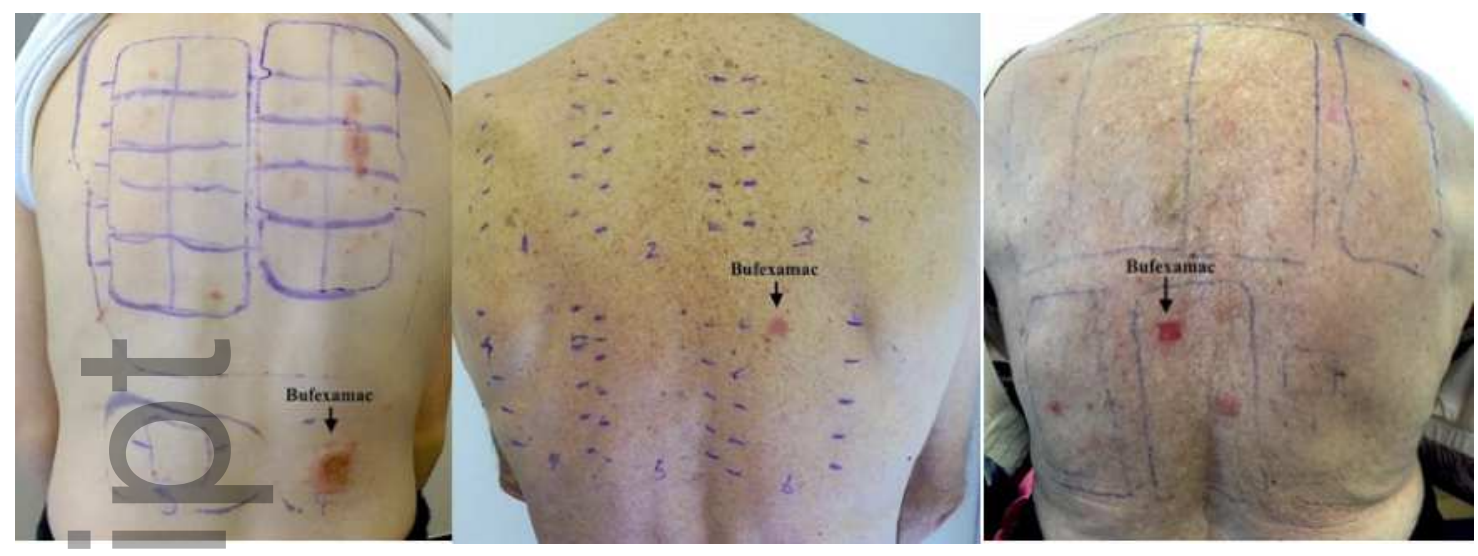

ajd_12910_f3.jpg

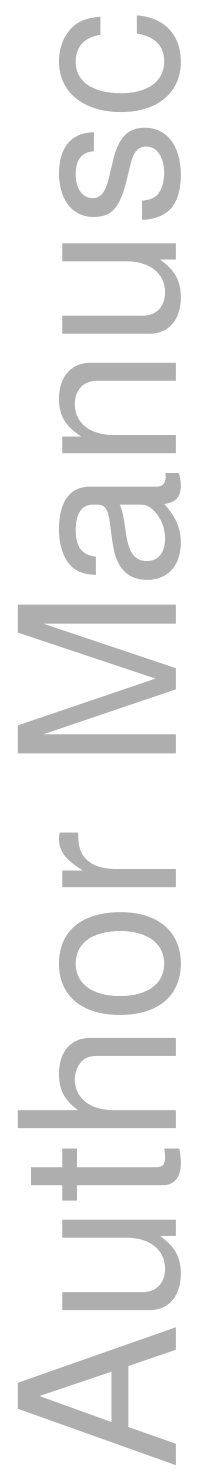

This article is protected by copyright. All rights reserved 


\section{University Library}

\section{- M I N E R VA}

\section{A gateway to Melbourne's research publications}

Minerva Access is the Institutional Repository of The University of Melbourne

\section{Author/s:}

Harris, AG;Saikal, SL;Scurry, J;Relic, J;Nixon, RL;Chee, P

Title:

Severe cutaneous eruptions following the topical use of preparations containing bufexamac: Is it time to reconsider its registration in Australia?

\section{Date:}

2019-02-01

\section{Citation:}

Harris, A. G., Saikal, S. L., Scurry, J., Relic, J., Nixon, R. L. \& Chee, P. (2019). Severe cutaneous eruptions following the topical use of preparations containing bufexamac: Is it time to reconsider its registration in Australia?. AUSTRALASIAN JOURNAL OF DERMATOLOGY, 60 (1), pp.53-56. https://doi.org/10.1111/ajd.12910.

Persistent Link:

http://hdl.handle.net/11343/284401 